\title{
Lung Cancer with a Small Cell Carcinoma Component Diagnosed from Pleural Effusion and a Squamous Cell Carcinoma Component Diagnosed from the Tumor
}

\author{
Yoshiaki Zaizen ${ }^{1}$, Takaaki Tokito ${ }^{1}$, Ken Masuda ${ }^{1}$, Koichi Azuma ${ }^{1}$, Yoshiki Naito ${ }^{2}$, \\ Shingo Tsuneyoshi ${ }^{1}$, Yuki Sakazaki ${ }^{1}$, Norikazu Matsuo ${ }^{1}$, Hidenobu Ishii ${ }^{1}$, Kazuhiko Yamada ${ }^{1}$, \\ Jun Akiba $^{2}$ and Tomoaki Hoshino ${ }^{1}$
}

\begin{abstract}
:
There have been few reports on the accuracy of the diagnosis of small-cell carcinoma based on a cytological examination of malignant pleural effusion, so whether or not such a diagnosis is possible using this approach alone remains unclear. We herein report a 76-year-old Japanese man in whom small-cell carcinoma was diagnosed cytopathologically from pleural effusion and squamous cell carcinoma was diagnosed histopathologically from a transbronchial biopsy. Tumor shrinkage was achieved by treatment with docetaxel, but the efficacy of carboplatin plus etoposide was inadequate. If small-cell carcinoma is detected on the basis of pleural fluid cytopathology alone, it is extremely important to perform a histopathological examination to rule out the possibility of other malignancies.
\end{abstract}

Key words: non-small cell lung cancer, small cell carcinoma, cytology, malignant pleural effusion

(Intern Med 57: 3419-3422, 2018)

(DOI: 10.2169/internalmedicine.1200-18)

\section{Introduction}

Lung cancer is the second leading cause of exudative pleural effusion, occurring in $7-15 \%$ of lung cancer patients (1). Adenocarcinoma is the most common histologic type of lung cancer associated with malignant pleural effusion. Although patients with small cell lung cancer (SCLC) may also develop malignant pleural effusion, its reported incidence in such cases is only $2.7 \%$ (2).

In SCLC, the rate of concordance between histopathological and cytopathologic findings obtained from the primary lesion in SCLC is reported to be $100 \%(3,4)$, whereas that between histopathological findings from a needle aspiration biopsy and cytopathologic findings from pleural effusion is $92 \%$ (5). We herein report a case in which there was discordance between the cytopathologic findings from pleural ef- fusion and the histopathologic findings from a transbronchial biopsy (TBB).

\section{Case Report}

A 76-year-old Japanese man was admitted with exertional dyspnea and right chest pain, and a chest X-ray abnormality was noted by a primary care physician. A hematological examination revealed an elevated level of Pro gastrin-releasing peptide (ProGRP), but carcinoembryonic antigen (CEA) and cytokeratin subunit 19 fragment (CYFRA) were within normal limits. The accumulation of pleural fluid was noted on the right side. We therefore performed chest drainage and continued pleurodesis thereafter. Cytopathology of the pleural fluid demonstrated malignant cells with a high nucleocytoplasmic ratio. Immunohistochemical staining (IHC) revealed positivity for synaptophysin and CD56 and negativity

${ }^{1}$ Division of Respirology, Neurology, and Rheumatology, Department of Internal Medicine, Kurume University School of Medicine, Japan and ${ }^{2}$ Department of Diagnostic Pathology, Kurume University School of Medicine, Japan

Received: March 16, 2018; Accepted: April 9, 2018; Advance Publication by J-STAGE: July 6, 2018

Correspondence to Dr. Takaaki Tokito, tokitou_takaaki@kurume-u.ac.jp 


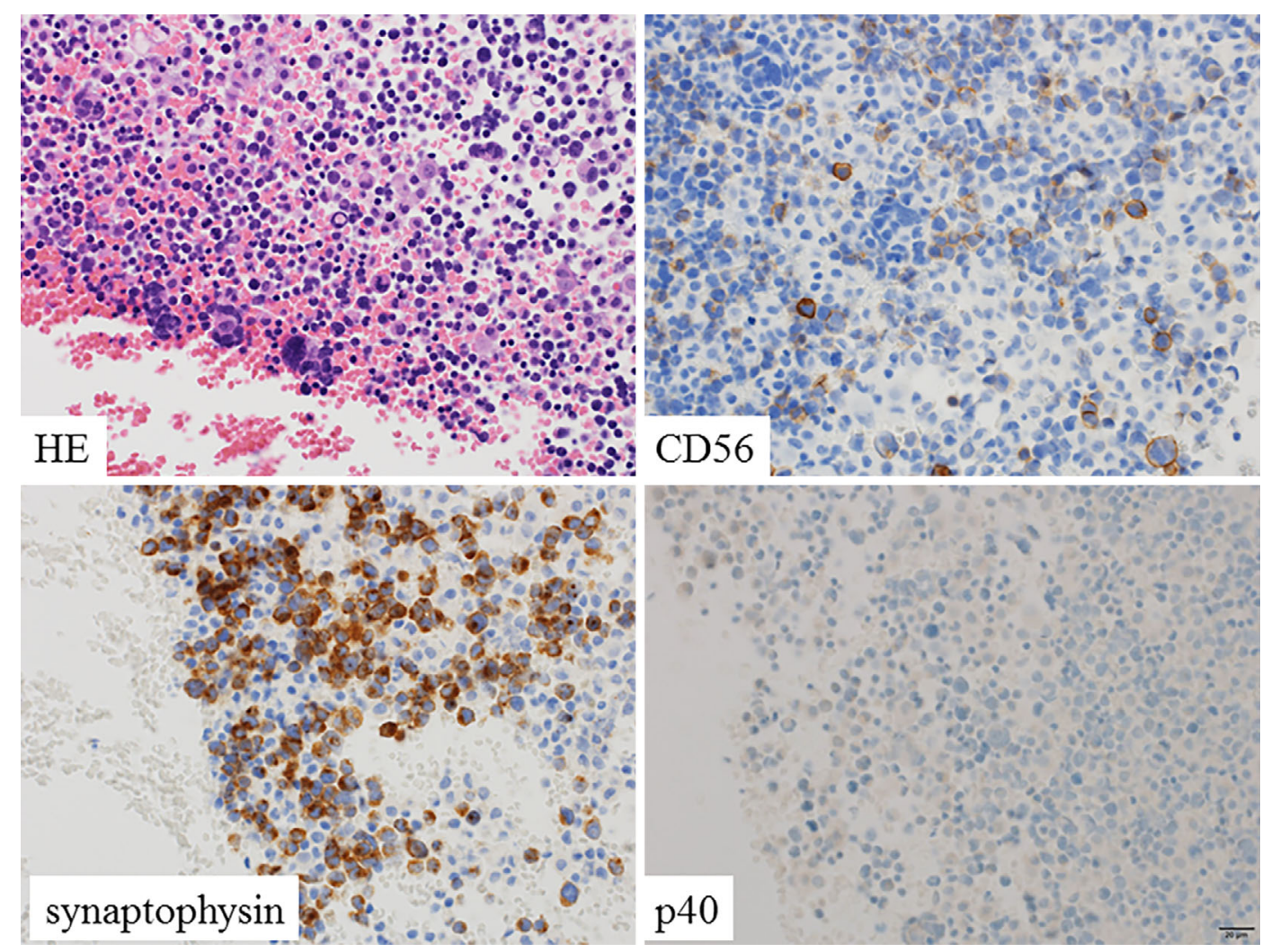

Figure 1. Pleural fluid cytopathology, showing tumor cells with a high nucleo-cytoplasmic ratio. Immunohistochemical staining showed positivity for synaptophysin and CD56 but negativity for p40.

for p40 (Fig. 1). On this basis, we diagnosed the patient with small cell carcinoma with cancerous peritonitis.

After chest drainage, systemic enhanced computed tomography revealed a tumor in the right upper lobe and mediastinal lymph node enlargement, with no primary lesion at other sites. A histopathological examination of a TBB specimen demonstrated malignant cells with cornification, and the cytological findings obtained by transbronchial brushing revealed necrosis. IHC demonstrated positivity for p40 and negativity for synaptophysin and CD56 (Fig. 2). On this basis, we diagnosed the lung tumor as squamous cell carcinoma. His carcinoma had a possibility of being SCLC combined with squamous cell carcinoma. However, the definition of combined SCLC is that the tumor includes a smallcell carcinoma component and non-small cell carcinoma component in the same specimen. Therefore, we could not confirm that his carcinoma was combined SCLC.

We considered that the small cell carcinoma component would determine the prognosis, so and the patient was given carboplatin plus etoposide as first-line chemotherapy. After receiving three courses of this regimen, an increase in both the primary tumor mass and the volume of pleural fluid was noted, and the regimen was considered to be ineffective. Docetaxel was therefore given as a second-line chemotherapy, which decreased the right hilar lymphadenopathy with atelectasis of the right upper lobe and dissemination, although the response was stable disease according to the Response Evaluation Criteria in Solid Tumors (RECIST). The right pleural effusion did not increase, and the tumor shrink- age continued for 6 months (Fig. 3).

\section{Discussion}

In the present patient, small cell carcinoma was diagnosed based on the findings of a cytopathologic examination of pleural effusion. However, no response was obtained with carboplatin plus etoposide. In a review by Schreiber et al., the accuracy of cytology for distinguishing SCLC from nonsmall cell lung cancer (NSCLC) was considered to be highbetween 0.94 and 1.00 (mean 0.98)-in a large cohort of 6,305 patients (6). However, in SCLC, Joanna et al. reported that the rate of concordance between the histopathological findings obtained from a needle aspiration biopsy and the cytopathologic findings obtained from pleural fluid was $92 \%$, although their study included only 12 cases (5). Another study reported that only $2.7 \%$ of 256 cases of small cell carcinoma were diagnosed from pleural effusion (2). The apparently lower reliability of a small cell carcinoma diagnosis based only on pleural effusion cytology may be attributable to the low number of cases analyzed.

We detected squamous cell carcinoma by a pathologic examination of a TBB sample from the primary tumor, and tumor shrinkage was achieved using docetaxel, with the response being sustained for six months. There is a general consensus that treatment for advanced tumors that include a small cell carcinoma component should be focused on SCLC, such as platinum plus etoposide, topotecan and amrubicin. However, whether or not there is a difference in the 


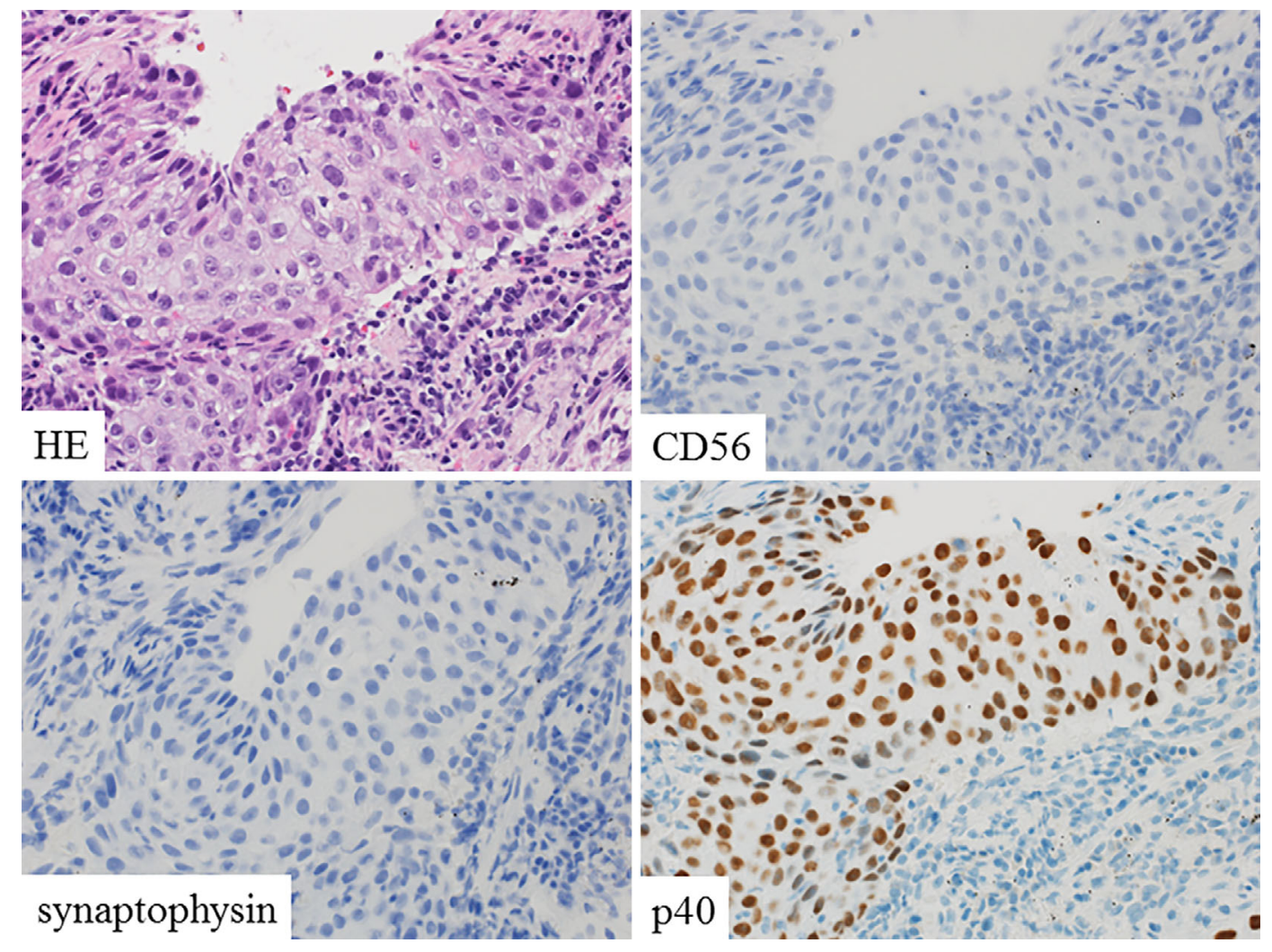

Figure 2. Histological findings of a transbronchial biopsy, showing cornification and necrosis. Immunohistochemical staining revealed positivity for p40 but negativity for synaptophysin and CD56.
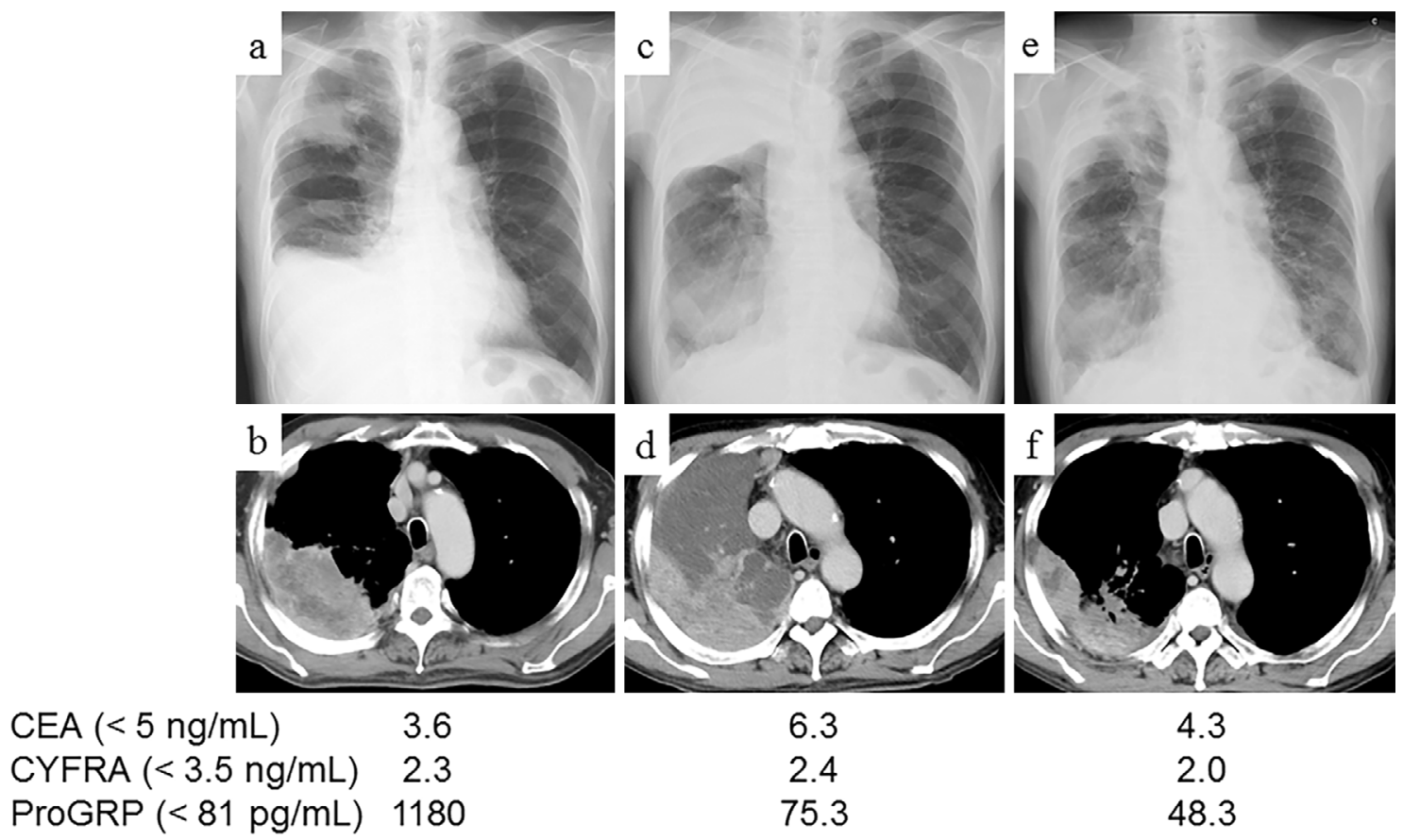

4.3

2.0

48.3

Figure 3. The course of radiographic findings and tumor markers. Chest X-ray (a) and CT (b) before first-line chemotherapy showed a lung tumor in the right upper lobe and right-side pleural effusion. (c) (d) The lung tumor in the right upper lobe increased in size after three courses of treatment with carboplatin plus etoposide. (e) (f) The lung tumor in the right upper lobe decreased in size after four courses of treatment with docetaxel.

degree of chemotherapeutic efficacy between the small cell carcinoma component and other combined histological com- ponents, such as adenocarcinoma and squamous cell carcinoma, is unclear. Further studies are warranted to determine 
the optimum treatment strategy for tumors with components of both SCLC and NSCLC.

In conclusion, we herein report a rare case in which small cell carcinoma was detected cytopathologically from pleural effusion and squamous cell carcinoma was detected histopathologically from a TBB sample. Chemotherapy with efficacy against SCLC did not elicit a clinical response, but tumor shrinkage was achieved with agents targeting NSCLC. If small cell carcinoma is detected on the basis of pleural effusion cytopathology alone, it is extremely important to perform a histopathological examination to rule out the possibility of other malignancies.

The authors state that they have no Conflict of Interest (COI).

\section{References}

1. Sahn SA. Malignancy metastatic to the pleura. Clin Chest Med 19: $351-361,1998$
2. David CC, Ellen CK, Herman TY, et al. Malignant pleural effusions due to small-cell lung carcinoma: a cytologic and immunocytochemical study. Diagn Cytopathol 25: 356-360, 2001.

3. Endo $\mathrm{T}$, Arakawa $\mathrm{M}$, Inoue $\mathrm{K}$, Inoue $\mathrm{S}$, Miyamoto $\mathrm{H}$, Abe $\mathrm{S}$. Comparison of histopathological and cytological diagnosis in lung cancer. J Jpn Soc Clin Cytol 20: 490-494, 1981.

4. Yanagawa N, Shiono S, Abiko M, et al. Histological typing accuracy rate of preoperative bronchoscopic cytology in patients with surgically resected lung carcinoma. Jpn J Lung Cancer 53: 220226, 2013.

5. Joanna DK, Barbara G, Rafal K, Sylia M, Ryszarda C. The value of cytological diagnosis of small cell lung carcinoma. Pneumonol Allergol Pol 78: 203-210, 2010.

6. Schreiber G, McCrory DC. Performance characteristics of different modalities for diagnosis of suspected lung cancer: summary of published evidence. Chest 123: 115S-128S, 2003.

The Internal Medicine is an Open Access journal distributed under the Creative Commons Attribution-NonCommercial-NoDerivatives 4.0 International License. To view the details of this license, please visit (https://creativecommons.org/licenses/ by-nc-nd/4.0/).

(C) 2018 The Japanese Society of Internal Medicine Intern Med 57: 3419-3422, 2018 